\title{
Study of Seasonal and Regional Prevalence of Norovirus in Children: A Step Towards Disease Control
}

\author{
Rishi Bolia ${ }^{1} \cdot$ Anshu Srivastava ${ }^{2}$ (D) \\ Received: 16 February 2021 / Accepted: 25 February 2021 / Published online: 6 March 2021 \\ (C) Dr. K C Chaudhuri Foundation 2021
}

In this issue of the Indian Journal of Pediatrics, Dr. Barsoum has studied the seasonal trend and disease severity of norovirus (Noro $\mathrm{V}$ ) infection in children $<3$ y of age presenting to a regional hospital in the town of Enniskillen, Northern Ireland [1]. One hundred fifty children who either presented to their emergency or were admitted with vomiting and/or diarrhea over a 1-y period were studied and $18(12 \%)$ were found to have Noro V infection. All Noro V patients had moderate or severe diarrhea and genogroup II was most common, seen in $16 / 18$ (89\%) patients. Double infection was present in $22 \%$ $(4 / 18)$ cases with astrovirus and Noro V together in 2 cases. There were three peak seasons of noroviral gastroenteritis (GE) during the year, between September and March. The fifth week of January witnessed the highest number of Noro $\mathrm{V}$ infections.

Noro V is an important cause of acute diarrhea with a world-wide pooled prevalence of $18 \%$ and has emerged as the most important cause of acute diarrhea in countries where the rotavirus vaccination has been introduced $[2,3]$. However, rotavirus diarrhea has increased from $15.8 \%$ in 2014 to $25 \%$ in 2016-17, while norovirus has remained constant at $13.2 \%$ vs $12 \%$ in the current study. This is difficult to explain without information about rotavirus vaccination rate in the area. It is also unclear why the authors chose to study only children $<3$ $y$ of age.

Anshu Srivastava

avanianshu@yahoo.com

1 Division of Pediatric Gastroenterology, Department of Pediatrics, All India Institute of Medical Sciences, Rishikesh 249203, Uttarakhand, India

2 Department of Pediatric Gastroenterology, Sanjay Gandhi Postgraduate Institute of Medical Sciences, Lucknow, Uttar Pradesh 226014, India
Noro V is often referred to as the "winter vomiting disease" and in a meta-analysis of 29 outbreak datasets, approximately three-quarters of all outbreaks occurred in the cool months [4]. Worldwide, genogroup II genotype 4 (GII.4) viruses predominate [3]. New GII. 4 variants emerge every $2-4$ y under positive selective pressure (immune escape variants). The emergence of such variants has been shown to be associated with a substantial increase in cases worldwide. Most cases of norovirus diarrhea are mild and can be managed as an outpatient only [3]. This is in contrast to the current study, which may be due to the selection criteria as milder cases may not be brought to the hospital. Noro V was not seen as a cause in cases with nosocomial diarrhea, but there is no information about the number of cases with nosocomial diarrhea which were enrolled for etiology testing. Although the study is limited by the number of cases, a comparative description of disease severity between single vs dual infection would have been useful.

A recent meta-analysis involving 148,867 cases with GE has shown that prevalence of Noro $\mathrm{V}$ is directly proportional to the economic strata of the country (18\% for upper middleincome vs 6\% for low-income countries) [5]. Despite the lower prevalence, developing countries account for approximately $99 \%$ of the 212,000 annual deaths caused by norovirus [6]. This highlights the importance of a dual-pronged strategy for reduction of diarrhea-related morbidity and mortality across the globe. First is to study the regional and seasonal etiological trends and target appropriate etiology-specific preventive measures such as environmental disinfection and effective vaccines. The second step is general, etiology-independent measures, like improved sanitation and hand hygiene, education of parents and caregivers, and prompt delivery of rehydration therapy, as they will work across all causes.

\section{Declarations}

Conflict of Interest None. 


\section{References}

1. Barsoum Z. Pediatric norovirus gastroenteritis in Ireland: seasonal trends, correlation with disease severity, nosocomial acquisition and viral co-infection. Indian J Pediatr. 2020. https://doi.org/10.1007/ s12098-020-03540-4.

2. McAtee CL, Webman R, Gilman RH, et al. Burden of norovirus and rotavirus in children after rotavirus vaccine introduction, Cochabamba, Bolivia. Am J Trop Med Hyg. 2016;94(1):212-7.

3. Ahmed SM, Hall AJ, Robinson AE, et al. Global prevalence of norovirus in cases of gastroenteritis: a systematic review and metaanalysis. Lancet Infect Dis. 2014;14(8):725-30.

4. Ahmed SM, Lopman BA, Levy K. A systematic review and metaanalysis of the global seasonality of norovirus. PLoS One. 2013;8(10):e75922. https://doi.org/10.1371/journal.pone.0075922.
5. Nguyen GT, Phan K, Teng I, Pu J, Watanabe T. A systematic review and meta-analysis of the prevalence of norovirus in cases of gastroenteritis in developing countries. Medicine (Baltimore). 2017;96(40):e8139. https://doi.org/10.1097/MD. 0000000000008139.

6. Pires SM, Fischer-Walker CL, Lanata CF, et al. Aetiology-specific estimates of the global and regional incidence and mortality of diarrhoeal diseases commonly transmitted through food. PLoS One. 2015;10(12):e0142927.

Publisher's Note Springer Nature remains neutral with regard to jurisdictional claims in published maps and institutional affiliations. 